\title{
Физика ядрА
}

\section{И ЭЛЕМЕНТАРНЫХ ЧАСТИЦ}

\author{
Atomic nucleus
}

AND ELEMENTARY PARTICLE PHYSICS

УДК 539.12.01

\section{НЕПЕРТУРБАТИВНАЯ КВАНТОВАЯ ХРОМОАИНАМИКА И КОРОТКИЕ СТРУНЫ В АННИГИАЯЦИИ ЭАЕКТРОН-ПОЗИТРОННОЙ ПАРЫ В ААРОНЫ}

\author{
М. Е. КОЖЕВНИКОВА ${ }^{1)}$, А. Г. ОГАНЕСЯН ${ }^{2)}$, О. В. ТЕРЯЕВ ${ }^{1)}$ \\ ${ }^{1)}$ Объединенный институт ядерных исследований, \\ ул. Жолио Кюри, 6, 141980, г. Дубна, Московская область, Россия \\ ${ }^{2)}$ Институт теоретической и экспериментальной физики им. А. И. Алиханова \\ Национального исследовательского изентра «Курчатовский институт», \\ ул. Большая Черемушкинская, 25, 117218, г. Москва, Россия
}

Разработан новый математический метод изучения непертурбативных поправок квантовой хромодинамики для процессов при малых энергиях. Используются обычная и аналитическая теория возмущений. Метод применим для извлечения конденсатов низших размерностей из функции Адлера, построенной на данных по $\mathrm{e}^{+} \mathrm{e}^{-}$-аннигиляции в пионные каналы: $\mathrm{e}^{+} \mathrm{e}^{-} \rightarrow \pi^{+} \pi^{-}, \mathrm{e}^{+} \mathrm{e}^{-} \rightarrow 2 \pi^{+} 2 \pi^{-}, \mathrm{e}^{+} \mathrm{e}^{-} \rightarrow \pi^{+} \pi^{-} 2 \pi^{0}, \mathrm{e}^{+} \mathrm{e}^{-} \rightarrow 3 \pi^{+} 3 \pi^{-}$и $\mathrm{e}^{+} \mathrm{e}^{-} \rightarrow 2 \pi^{+} 2 \pi^{-} 2 \pi^{0}$.

\section{Образец цитирования:}

Кожевникова МЕ, Оганесян АГ, Теряев ОВ. Непертурбативная квантовая хромодинамика и короткие струны в аннигиляции электрон-позитронной пары в адроны. Журнал Белорусского государственного университета. Физика. 2019; $2: 4-15$.

https://doi.org/10.33581/2520-2243-2019-2-4-15

\section{For citation:}

Kozhevnikova ME, Oganesian AG, Teryaev OV. Non-perturbative quantum chromodynamics and short strings in electronpositron pair annihilation to hadrons. Journal of the Belarusian State University. Physics. 2019;2:4-15. Russian. https://doi.org/10.33581/2520-2243-2019-2-4-15

\section{Ав торы:}

Марина Евгеньевна Кожевникова - младший научный сотрудник научно-экспериментального отдела физики столкновений тяжелых ионов на комплексе NICA лаборатории физики высоких энергий им. В. И. Векслера и А. М. Балдина.

Армен Гургенович Оганесян - кандидат физико-математических наук; старший научный сотрудник лаборатории теории сильных взаимодействий.

Олег Валерьянович Теряев - доктор физико-математических наук; начальник сектора лаборатории теоретической физики им. Н. Н. Боголюбова.

\footnotetext{
Authors:

Marina E. Kozhevnikova, junior researcher at the division of physics of the collisions of heavy ions on the NICA complex, Veksler and Baldin laboratory of high energy physics.

kozhevnikova@jinr.ru

Armen G. Oganesian, $\mathrm{PhD}$ (physics and mathematics); senior researcher at the laboratory of strong interaction theory. armen@itep.ru

Oleg V. Teryaev, doctor of science (physics and mathematics); head at the Bogoliubov laboratory of theoretical physics group. teryaev@theor.jinr.ru
} 
Получены высокоточные фиты данных. Особое внимание уделяется вопросу: существует ли вклад оператора размерности 2? Указанная функция Адлера исследована в зависимости как от $Q$, так и от параметра $M^{2}$ преобразования Бореля. Показано, что в рамках предложенного метода коэффициент $C_{2}$ при операторе размерности 2 отрицателен, его совместимость с нулем зависит от выбора аналитической или обычной теории возмущений, от интервала, на котором рассматривается борелизованная функция Адлера, и от величины $\Lambda$. Существование ненулевого оператора размерности 2 может быть объяснено эффектом коротких струн.

Ключевые слова: квантовая хромодинамика; теория возмущений; дисперсионные соотношения; преобразование Бореля; рождение адронов в $\mathrm{e}^{+} \mathrm{e}^{-}$-аннигиляции; непертурбативные поправки.

Благодарность. Выражаем благодарность коллегам доктору физико-математических наук О. П. Соловцовой, Д. В. Дедовичу и доктору физико-математических наук А. В. Сидорову за ценные замечания и обсуждения. Исследование частично поддержано Объединенным институтом ядерных исследований и Белорусским республиканским фондом фундаментальных исследований (грант № F18D-002).

\title{
NON-PERTURBATIVE QUANTUM CHROMODYNAMICS AND SHORT STRINGS IN ELECTRON-POSITRON PAIR ANNIHILATION TO HADRONS
}

\author{
M. E. KOZHEVNIKOVA, A. G. OGANESIAN ${ }^{\mathrm{b}}$, O. V.TERYAEV \\ ${ }^{\text {a } J o i n t ~ I n s t i t u t e ~ f o r ~ N u c l e a r ~ R e s e a r c h, ~}$ \\ 6 Zholio Kyuri Street, Dubna 141980, Moscow region, Russia \\ ${ }^{\mathrm{b}}$ Institute of Theoretical and Experimental Physics, \\ 25 Bol'shaya Cheremushkinskaya Street, Moscow 117218, Russia \\ Corresponding author: M. E. Kozhevnikova (kozhevnikova@jinr.ru)
}

We develop a new mathematical method to study the non-perturbative quantum chromodynamics effects in lowenergy processes using ordinary and analytic perturbation theory (PT and APT). We apply this tool to extract the lowest dimensional condensates from the high-precision fits of the data on $\mathrm{e}^{+} \mathrm{e}^{-}$-annihilation to pion channels: $\mathrm{e}^{+} \mathrm{e}^{-} \rightarrow \pi^{+} \pi^{-}$, $\mathrm{e}^{+} \mathrm{e}^{-} \rightarrow 2 \pi^{+} 2 \pi^{-}, \mathrm{e}^{+} \mathrm{e}^{-} \rightarrow \pi^{+} \pi^{-} 2 \pi^{0}, \mathrm{e}^{+} \mathrm{e}^{-} \rightarrow 3 \pi^{+} 3 \pi^{-}$and $\mathrm{e}^{+} \mathrm{e}^{-} \rightarrow 2 \pi^{+} 2 \pi^{-} 2 \pi^{0}$. The special attention is devoted to the existence of the contribution of dimension 2 operator. Our consideration is based on the dependence of Adler function corresponding to $\mathrm{e}^{+} \mathrm{e}^{-}$-annihilation into pions on both $Q$ and Borel transform parameter $M^{2}$. It is shown that within the framework of the proposed method the $C_{2}$ coefficient of the dimension 2 operator is negative and is closer to zero for analytic perturbation theory, which may therefore be partially responsible for the effect of short strings.

Keywords: quantum chromodynamics; perturbation theory; dispersion relations; Borel transform; hadron production in $\mathrm{e}^{+} \mathrm{e}^{-}$-annihilation; non-perturbative corrections.

Acknowledgements. It is a pleasure to thank doctor of science O. P. Solovtsova, D. V. Dedovich and doctor of science A. V. Sidorov for discussions and interest in this work. This research was supported in part by the Joint Institute for Nuclear Research and Belarusian Republic Foundation for Fundamental Research (grant No. F18D-002).

\section{Введение}

Вопрос о существовании оператора размерности 2 , вклад которого в правила сумм $[1 ; 2]$ квантовой хромодинамики (КХД) пропорционален $\frac{1}{Q^{2}}$, а также свойства высших поправок операторного разложения изучаются уже длительное время [3-5]. В основополагающей работе В. И. Захарова и соавторов в 1999 г. была предложена концепция коротких струн, ведущих к поправкам размерности 2 в операторном разложении [3].

Так, в корнельском потенциале [5], выражаемом формулой

$$
V(r) \approx-\frac{4 \alpha_{s}(r)}{3 r}+k r
$$


второй член $k r$ описывает потенциал струны и связан с явлением конфайнмента. В случае его применения на малых расстояниях (короткая струна) он приводит к поправкам вида $\frac{k}{Q^{2}}$. Заметим, что в локальном операторном разложении такая поправка отсутствует, а имеющаяся первая поправка имеет вид $\sim \frac{\left\langle G_{\mu v} G^{\mu v}\right\rangle}{Q^{4}}$.

В статье [4] установлена корреляция между короткой струной и порядком теории возмущений (ТВ). Это аналогично взаимосвязи между порядком ТВ и высшим твистом в глубоко неупругом рассеянии $[7 ; 8]$. Вклад оператора размерности 2 в процессах е $\mathrm{e}^{+}-$-аннигиляции исследовался ранее в [5; 9], где было показано, что он совместим с нулем и имеет большие ошибки.

Настоящий анализ основан на использовании функции Адлера ( $D$-функции), являющейся простым двухточечным коррелятором, удобным для сравнения с экспериментальными данными [10]. Мы изучаем как ее зависимость от $Q$, так и преобразование Бореля от нее во взаимосвязи с соответствующим параметром $M^{2}$. При использовании последнего вклад лидирующего порядка ТВ обращается в нуль, а вклад поправок может быть изучен точнее.

Помимо обычной ТВ в КХД существует аналитический подход, называемый аналитической теорией возмущений (ATB) $[11 ; 12]$, которая включает в себя базовые принципы локальной квантовой теории поля, в простейшем случае выражающиеся в $Q^{2}$-аналитичности Челлена - Лемана (см. обзор [13]).

В рамках ATB аналитическая «бегущая константа» связи (эффективный заряд) не имеет нефизических сингулярностей, и переход из евклидовой области в область Минковского оказывается самосогласованным [14]. В асимптотической области при больших $Q^{2}$ подходы ТВ и АТВ совпадают.

Список применений АТВ, представленный в обзоре [13], может быть дополнен применением в КХДанализе глубоко неупругого рассеяния [8; 15-17].

Основная цель работы - проверить, проявляется ли в данных вклад оператора размерности 2. Для этого построена модель экспериментальных данных по $\mathrm{e}^{+} \mathrm{e}^{-}$-аннигиляции в пионные каналы: $\mathrm{e}^{+} \mathrm{e}^{-} \rightarrow \pi^{+} \pi^{-}$, $\mathrm{e}^{+} \mathrm{e}^{-} \rightarrow 2 \pi^{+} 2 \pi^{-}, \mathrm{e}^{+} \mathrm{e}^{-} \rightarrow \pi^{+} \pi^{-} 2 \pi^{0}, \mathrm{e}^{+} \mathrm{e}^{-} \rightarrow 3 \pi^{+} 3 \pi^{-}$и $\mathrm{e}^{+} \mathrm{e}^{-} \rightarrow 2 \pi^{+} 2 \pi^{-} 2 \pi^{0}$. Далее с использованием указанной модели строится функция Адлера в дисперсионной форме, применяется преобразование Бореля, составляются правила сумм и извлекаются коэффициенты операторного разложения.

\section{Фитирование данных}

Данные представлены наборами экспериментальных точек, и для их анализа предпочтительно использовать аналитические фиты, являющиеся интегрируемыми функциями. Поскольку имеются данные по различным каналам аннигиляции, полученные на различных детекторах, каждый фит производится отдельно.

Проводимый анализ включает в себя данные по следующим каналам по $\mathrm{e}^{+} \mathrm{e}^{-}$-аннигиляции:

$$
\begin{aligned}
& \mathrm{e}^{+} \mathrm{e}^{-} \rightarrow \pi^{+} \pi^{-}[18], \\
& \mathrm{e}^{+} \mathrm{e}^{-} \rightarrow 2 \pi^{+} 2 \pi^{-}[19], \\
& \mathrm{e}^{+} \mathrm{e}^{-} \rightarrow \pi^{+} \pi^{-} 2 \pi^{0}[20-25], \mathrm{e}^{+} \mathrm{e}^{-} \rightarrow 3 \pi^{+} 3 \pi^{-}[26], \\
& \mathrm{e}^{+} \mathrm{e}^{-} \rightarrow 2 \pi^{+} 2 \pi^{-} 2 \pi^{0}[26] .
\end{aligned}
$$

Для описания набора экспериментальных точек $\left\{\left(s_{i}, f_{\exp }\left(s_{i}\right)\right)\right\}_{i=1, \ldots, N}$ подбирается функция $f_{\mathrm{th}}\left(s ;\left\{a_{1}, \ldots, a_{d}\right\}\right)$, коэффициенты которой определяются минимизацией функционала $\chi^{2}$ :

$$
\chi^{2}\left(a_{1}, \ldots, a_{d}\right)=\frac{1}{N_{\text {d.f }}} \sum_{n=1}^{N} \frac{\left(f_{\text {exp }}\left(s_{n}\right)-f_{\text {th }}\left(s_{n} ;\left\{a_{1}, \ldots, a_{d}\right\}\right)\right)^{2}}{\delta f_{\text {exp }}\left(s_{n}\right)^{2}},
$$

где $N_{\text {d.f }}=N-d$ есть число степеней свободы в процедуре фитирования, $N$ - число экспериментальных точек, $d$ - число параметров фитирования; $\delta f_{\text {ехр }}\left(s_{n}\right)$ - статистическая ошибка точки $n$. Качество фитирующей процедуры определяется минимальным значением функционала $\chi^{2}$.

Данные для процесса (1) представлены в виде квадрата модуля формфактора $\left|F_{\pi}(s)\right|^{2}$ в зависимости от энергии $\sqrt{s}$. Для фитирования используется трехрезонансная модель Гунариса - Сакураи [18; 27], формфактор каждого векторного мезонного резонанса $V\left(V=\rho, \omega, \rho^{\prime}\right)$ вычисляется по формуле Брейта Вигнера: 


$$
F^{\mathrm{BW}}\left(s, m_{V}, \Gamma_{V}\right)=\frac{m_{V}^{2}\left(1+d \cdot \Gamma_{V} / m_{V}\right)}{m_{V}^{2}-s+f\left(s, m_{V}, \Gamma_{V}\right)-i m_{V} \Gamma_{V}(s)},
$$

где

$$
\begin{gathered}
\Gamma_{V}(s)=\Gamma_{V}\left(\frac{k(s)}{k\left(m_{V}^{2}\right)}\right)^{3}, k(s)=\frac{\sqrt{s-4 m_{\pi}^{2}}}{2} ; \\
f\left(s, m_{V}, \Gamma_{V}\right)=\Gamma_{V} \frac{m_{V}^{2}}{k\left(m_{V}^{2}\right)^{3}}\left[k^{2}(s)\left(h(s)-h\left(m_{V}^{2}\right)\right)-\left(s-m_{V}^{2}\right) k^{2}\left(m_{V}^{2}\right) h^{\prime}\left(m_{V}^{2}\right)\right], \\
h(s)=\frac{2}{\pi} \frac{k(s)}{\sqrt{s}} \ln \left(\frac{\sqrt{s}+2 m_{\pi}}{2 m_{\pi}}\right), h^{\prime}\left(m_{V}^{2}\right)=\left.h^{\prime}(s)\right|_{s=m_{V}^{2}} .
\end{gathered}
$$

Условие нормировки $F^{\mathrm{Bw}}\left(0, m_{V}, \Gamma_{V}\right)=1$ выполняется автоматически.

Полный пионный формфактор имеет вид

$$
F_{\pi}\left(s,\left\{\rho, \omega, \rho^{\prime}\right\}\right)=\frac{F_{\pi}(s,\{\rho, \omega\})+\alpha_{\rho^{\prime}} F^{\mathrm{BW}}\left(s, m_{\rho^{\prime}}, \Gamma_{\rho^{\prime}}\right)}{1+\alpha_{\rho^{\prime}}},
$$

где

$$
F_{\pi}(s,\{\rho, \omega\})=F^{\mathrm{BW}}\left(s, m_{\rho}, \Gamma_{\rho}\right) \frac{1+\alpha_{\omega} F^{\mathrm{BW}}\left(s, m_{\omega}, \Gamma_{\omega}\right)}{1+\alpha_{\omega}} .
$$

Лучший фит характеризуется значением $\chi^{2}=1,05$.

Сечение в нанобарнах (нбн) пересчитывается из формфактора по стандартной формуле

$$
\sigma(s)=|F(s)|^{2} \frac{\pi \alpha_{\mathrm{em}}}{3 s}\left(1-\frac{4 m_{\pi}^{2}}{s}\right)^{3 / 2} 0,3894 \cdot 10^{6},
$$

\begin{tabular}{|c|c|c|c|c|}
\hline V & $m_{V}$, ГэВ & $\Gamma_{V}, \Gamma э \mathrm{~B}$ & $\alpha_{V}$ & $d$ \\
\hline$\rho$ & $0,775^{*}$ & $0,148 \pm 0,002$ & 1 & \multirow{3}{*}{$0,408 \pm 0,041$} \\
\hline$\omega$ & $0,782^{*}$ & $0,008^{*}$ & $0,0020 \pm 0,0003$ & \\
\hline$\rho^{\prime}$ & $1,353 \pm 0,024$ & $0,328 \pm 0,149$ & $-0,085 \mp 0,005$ & \\
\hline
\end{tabular}

где $\alpha_{\mathrm{em}}=\frac{1}{137}-$ константа электромагнитного взаимодействия.

Результаты фитирования показаны в табл. 1.

Таблица 1

Результаты фитирования для процесса $\mathrm{e}^{+} \mathrm{e}^{-} \rightarrow \pi^{+} \pi^{-}, \chi_{\min }^{2}=1,05$

Table 1

The fitting results for the process $\mathrm{e}^{+} \mathrm{e}^{-} \rightarrow \pi^{+} \pi^{-}, \chi_{\min }^{2}=1.05$

*3начения зафиксированы и взяты из PDG [26].

Для сечений процессов (2)-(4) введем модель формфактора в виде суммы гауссовых кривых, описывающих широкие резонансы:

$$
F_{\text {Gauss }}\left(s,\left\{M_{i}, \sigma_{i}, \alpha_{i}\right\}\right)=\sum_{i=1}^{3} \alpha_{i} e^{-\left(\sqrt{s}-M_{i}\right)^{2} /\left(2 \sigma_{i}^{2}\right)} .
$$

Результаты представлены в табл. 2. 
Результаты фитирования для различных процессов $\mathrm{e}^{+} \mathrm{e}^{-}$-аннигиляции

The fitting results for particular $\mathrm{e}^{+} \mathrm{e}^{-}$-annihilation channels

\begin{tabular}{|c|c|c|c|c|}
\hline Процесс & $i$ & $M_{i}$, ГэВ & $\sigma_{i}$, ГэВ & $\alpha_{i}$ \\
\hline \multirow{2}{*}{$\mathrm{e}^{+} \mathrm{e}^{-} \rightarrow 2 \pi^{+} 2 \pi^{-}}$, & 1 & $1,512 \pm 0,003$ & $0,242 \pm 0,002$ & $1,560 \pm 0,016$ \\
\cline { 2 - 5 }$\chi_{\min }^{2}=1,85$ & 2 & $2,125 \pm 0,016$ & $0,231 \pm 0,012$ & $0,458 \pm 0,022$ \\
\cline { 2 - 5 } & 3 & $2,656 \pm 0,025$ & $0,808 \pm 0,019$ & $0,590 \pm 0,014$ \\
\hline \multirow{2}{*}{$\mathrm{e}^{+} \mathrm{e}^{-} \rightarrow \pi^{+} \pi^{-} 2 \pi^{0}}$, & 1 & $1,786 \pm 0,005$ & $0,327 \pm 0,003$ & $1,484 \pm 0,029$ \\
\cline { 2 - 5 }$\chi_{\min }^{2}=8,45$ & 2 & $1,070 \pm 0,006$ & $0,099 \pm 0,005$ & $0,370 \pm 0,017$ \\
\cline { 2 - 5 } & 3 & $1,343 \pm 0,004$ & $0,188 \pm 0,004$ & $0,916 \pm 0,012$ \\
\hline $\mathrm{e}^{+} \mathrm{e}^{-} \rightarrow 3 \pi^{+} 3 \pi^{-}$, & 1 & $1,789 \pm 0,015$ & $0,083 \pm 0,012$ & $0,154 \pm 0,020$ \\
\cline { 2 - 5 }$\chi_{\min }^{2}=0,62$ & 2 & $2,050 \pm 0,014$ & $0,291 \pm 0,011$ & $0,433 \pm 0,017$ \\
\hline \multirow{2}{*}{$\mathrm{e}^{+} \mathrm{e}^{-} \rightarrow 2 \pi^{+} 2 \pi^{-} 2 \pi^{0}}$, & 1 & $2,348 \pm 0,012$ & $0,331 \pm 0,008$ & $1,598 \pm 0,085$ \\
\cline { 2 - 5 }$\chi_{\min }^{2}=1,03$ & 2 & $1,740 \pm 0,011$ & $0,120 \pm 0,009$ & $0,558 \pm 0,035$ \\
\hline
\end{tabular}

При расчете $\chi^{2}$ использовались только статистические ошибки. Основной вклад в полную ошибку сечения вносит систематическая ошибка, которая будет учтена ниже, а вклад статистических ошибок пропорционален $\frac{1}{N_{\text {d.f }}}$ и пренебрежимо мал при большом количестве точек.

Ошибка полного сечения вычисляется по формуле

где $j$ - номер канала.

$$
\Delta \sigma(s)=\sqrt{\sum_{j} \Delta \sigma_{j}^{2}(s)}
$$

\section{Вычисление $\boldsymbol{R}$-отношения и функции Адлера}

По определению $R$-отношение - это отношение сечения $\mathrm{e}^{+} \mathrm{e}^{-}$-аннигиляции в адроны к сечению $\mathrm{e}^{+} \mathrm{e}^{-}-$ аннигиляции в пару мюонов:

где

$$
R(s)=\frac{\sigma_{\mathrm{e}^{+} \mathrm{e}^{-} \rightarrow \text { hadrons }}(s)}{\sigma_{\mathrm{e}^{+} \mathrm{e}^{-} \rightarrow \mu^{+} \mu^{-}}(s)},
$$

$$
\sigma_{\mathrm{e}^{+} \mathrm{e}^{-} \rightarrow \mu^{+} \mu^{-}}(s)=\frac{4 \pi \alpha_{\mathrm{em}}^{2}}{3 s} .
$$

Полное $R$-отношение - это сумма $R$-отношений отдельных процессов:

$$
R(s)=\sum_{i} R_{i}(s), R_{i}(s)=\frac{\sigma_{\mathrm{e}^{+} \mathrm{e}^{-} \rightarrow \text { hadrons of type } i}(s)}{\sigma_{\mathrm{e}^{+} \mathrm{e}^{-} \rightarrow \mu^{+} \mu^{-}}(s)} .
$$

Здесь суммирование проводится по процессам (1)-(5).

Теоретическая формула полного $R$-отношения в однопетлевом приближении в ТВ и АТВ имеет вид

$$
R_{\mathrm{th}}^{\mathrm{PT} / \mathrm{APT}}(s)=N_{c} \sum_{q} e_{q}^{2}\left(1+\frac{\alpha_{s}^{\mathrm{PT} / \mathrm{APT}}(s)}{\pi}\right)
$$

где $N_{c}=3 ; e_{q}-$ заряд кварка аромата $q$. Мы извлекаем ток $j_{\mu}=\frac{1}{2}\left(\bar{u} \gamma_{\mu} u-\bar{d} \gamma_{\mu} d\right)$ с изоспином $I=1$ из полного электромагнитного тока, заменяя фактор

$$
\sum_{q} e_{q}^{2} \rightarrow \frac{1}{4}+\frac{1}{4}=\frac{1}{2}
$$


Результат суммирования приведенных выше модельных вкладов представлен на рис. 1 черной сплошной линией.

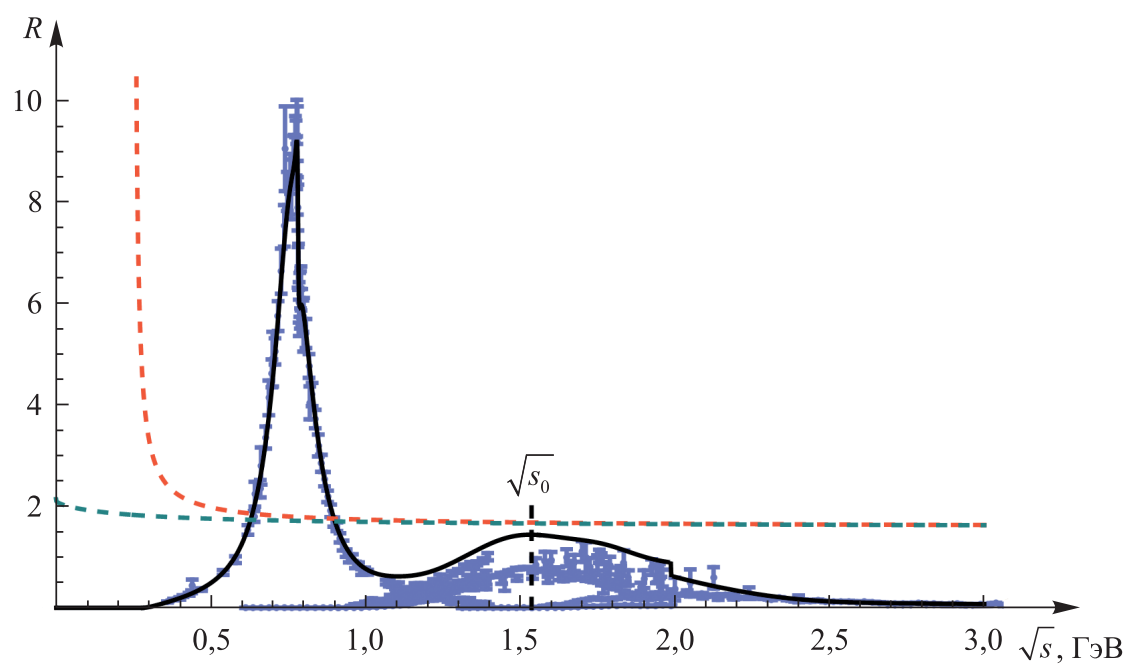

Puc. 1. Полное $R$-отношение в зависимости от $\sqrt{s}$ при $\sqrt{s} \leq 3$ ГэВ (черная кривая), экспериментальные точки (синие), теоретическая форма $R_{\mathrm{th}}$ в ТВ (красная пунктирная линия) и АТВ (голубая пунктирная линия). Порог континуума $s_{0} \approx 1,54^{2}$ ГэВ ${ }^{2}$

Fig. 1. The full $R$-ratio in dependence on $\sqrt{s}$ at $\sqrt{s} \leq 3 \mathrm{GeV}$ (black), the experimental data (blue dots), the theoretical representation $R_{\text {th }}$ in the PT (red, dashed) and in the APT (blue, dashed). The continuum threshold is $s_{0} \approx 1.54^{2} \mathrm{GeV}^{2}$

Ошибка $R$-отношения на интервале от порога $4 m_{\pi}^{2}$ до $s_{0}$ вычисляется через ошибку сечения:

$$
\Delta R(s)=\frac{\Delta \sigma_{\mathrm{e}^{+} \mathrm{e}^{-} \rightarrow \text { hadrons }}(s)}{\sigma_{\mathrm{e}^{+} \mathrm{e}^{-} \rightarrow \mu^{+} \mu^{-}}(s)} .
$$

Существуют два способа записи $D$-функции. Первый является дисперсионным и имеет вид

$$
D_{\exp }\left(Q^{2}\right)=Q^{2} \int_{4 m_{\pi}^{2}}^{\infty} \frac{R_{\text {exp-th }}(s) d s}{\left(s+Q^{2}\right)^{2}} .
$$

Второй способ включает операторное разложение в подходах ТВ и АТВ:

$$
\begin{gathered}
D_{\mathrm{PT}+\mathrm{OPE}}\left(Q^{2}\right)=\frac{3}{2}\left(1+\frac{\alpha_{s}\left(Q^{2}\right)}{\pi}+\sum_{n \geq 1} \Gamma(n) \frac{C_{2 n}}{Q^{2 n}}\right), \\
D_{\mathrm{APT}+\mathrm{OPE}}\left(Q^{2}\right)=\frac{3}{2}\left(1+\frac{\mathcal{A}_{s}\left(Q^{2}\right)}{\pi}+\sum_{n \geq 1} \Gamma(n) \frac{\tilde{C}_{2 n}}{Q^{2 n}}\right) .
\end{gathered}
$$

В качестве стандартного эффективного заряда используется однопетлевое приближение:

$$
\alpha_{s}\left(Q^{2}\right)=\frac{4 \pi}{\beta_{0}} \frac{1}{\ln \left(Q^{2} / \Lambda^{2}\right)} .
$$

Здесь $\beta_{0}=11-\frac{2 N_{f}}{3}$. Заметим, что в формуле (9) имеется нефизическая сингулярность при $Q^{2}=\Lambda^{2}$.

Эффективный заряд в рамках АТВ имеет вид

$$
\mathcal{A}_{s}\left(Q^{2}\right)=\frac{4 \pi}{\beta_{0}}\left[\frac{1}{\ln \left(Q^{2} / \Lambda_{\mathrm{APT}}^{2}\right)}-\frac{\Lambda_{\mathrm{APT}}^{2}}{Q^{2}-\Lambda_{\mathrm{APT}}^{2}}\right] .
$$

В выражении (10) дополнительный член исключает сингулярность. 


\section{Преобразование Бореля $D$-функции и правила сумм}

Преобразование Бореля от функции $f(x)$ по определению имеет следующий вид:

$$
\hat{B}_{x \rightarrow y}[f(x)]=\lim _{n \rightarrow \infty} \frac{(-x)^{n}}{\Gamma(n)}\left[\frac{d^{n}}{d x^{n}} f(x)\right]_{x=n y} .
$$

Применим его к функции Адлера. Преобразованные по Борелю выражения (6)-(8) запишутся в виде

$$
\begin{gathered}
\hat{B}_{Q^{2} \rightarrow M^{2}}\left[D_{\exp }\left(Q^{2}\right)\right]=\Phi_{\exp }\left(M^{2}\right)=\int_{4 m_{\pi}^{2}}^{\infty} R_{\exp -\text { th }}(s)\left(1-\frac{s}{M^{2}}\right) e^{-s / M^{2}} \frac{d s}{M^{2}}, \\
\hat{B}_{Q^{2} \rightarrow M^{2}}\left[D_{\mathrm{PT}+\mathrm{OPE}}\left(Q^{2}\right)\right]=\Phi_{\mathrm{PT}+\mathrm{OPE}}\left(M^{2}\right)=\frac{3}{2}\left(\frac{\hat{B}_{Q^{2} \rightarrow M^{2}}\left[\alpha_{s}\left(Q^{2}\right)\right]}{\pi}+\frac{C_{2}}{M^{2}}+\frac{C_{4}}{M^{4}}+\frac{C_{6}}{M^{6}}\right), \\
\hat{B}_{Q^{2} \rightarrow M^{2}}\left[D_{\mathrm{APT}+\mathrm{OPE}}\left(Q^{2}\right)\right]=\Phi_{\mathrm{APT}+\mathrm{OPE}}\left(M^{2}\right)=\frac{3}{2}\left(\frac{\hat{B}_{Q^{2} \rightarrow M^{2}}\left[\mathcal{A}_{s}\left(Q^{2}\right)\right]}{\pi}+\frac{\tilde{C}_{2}}{M^{2}}+\frac{\tilde{C}_{4}}{M^{4}}+\frac{\tilde{C}_{6}}{M^{6}}\right) .
\end{gathered}
$$

Приравнивание обеих форм функции Адлера приводит к правилам сумм для ТВ и АТВ:

$$
\Phi_{\text {exp }}\left(M^{2}\right)=\left\{\begin{array}{l}
\Phi_{\mathrm{PT}+\mathrm{OPE}}\left(M^{2}\right), \\
\Phi_{\mathrm{APT}+\mathrm{OPE}}\left(M^{2}\right) .
\end{array}\right.
$$

Для дальнейших вычислений найдем ошибку $\Delta \Phi_{\exp }\left(M^{2}\right)$ :

$$
\Delta \Phi_{\exp }\left(M^{2}\right)=\int_{4 m_{\pi}^{2}}^{\infty} \Delta R(s)\left(1-\frac{s}{M^{2}}\right) e^{-s / M^{2}} \frac{d s}{M^{2}} .
$$

\section{Анализ правил сумм и извлечение конденсатов}

Перейдем к обсуждению правил сумм (11). Сравним две формы преобразованной по Борелю функции Адлера с помощью минимизации $\chi^{2}$-функционала (варьируются коэффициенты $C_{2}$ и $C_{4}$ ):

$$
\chi^{2}\left(C_{2}, C_{4}\right)=\frac{1}{N_{\text {d.f }}} \sum_{n=1}^{N} \frac{\left(\Phi_{\text {exp }}\left(M_{n}^{2}\right)-\Phi_{\mathrm{PT}(\mathrm{APT})+\mathrm{OPE}}\left(M_{n}^{2} ;\left\{C_{2}, C_{4}\right\}\right)\right)^{2}}{\Delta \Phi_{\text {exp }}\left(M_{n}^{2}\right)^{2}},
$$

где $N_{\text {d.f }}=N-d$ есть число степеней свободы, $N$ - количество точек на выбранном интервале, $d$ - число варьируемых параметров (в данном случае $d=2$ ).

Отметим, что значение кваркового конденсата зафиксировано [29], и коэффициент $C_{6}$ равен:

$$
C_{6}=-\frac{448 \pi^{3}}{27} \alpha_{s}\langle\bar{q} q\rangle^{2} \approx-0,116 \text { Гэ } \mathrm{B}^{6} .
$$

Главной качественной особенностью результатов (табл. 3,4 и рис. 2, 3) является антикорреляция вклада короткой струны и глюонного конденсата, отмеченная ранее в нашей работе [30]. На рисунках для сравнения горизонтальными линиями отмечены известные значения глюонного конденсата $[1 ; 29 ; 31]$.

С помощью полученных данных проверим совместимость метода преобразования Бореля со стандартным анализом $D$-функции. Для этого построим зависимость функции Адлера от $Q$ с использованием параметров, полученных путем преобразования Бореля (12) в интервале по 0,5 Гэ $\mathrm{B}^{2} \leq M^{2} \leq 8$ Гэ $\mathrm{B}^{2}$ (рис. 4). Кривая, построенная с помощью операторного разложения, находится в разумном согласии с дисперсионной кривой при $Q>1$ ГэВ. 
Результаты фитирования на различных интервалах по $M^{2}$ в ТВ при $\Lambda=0,25$ ГэВ

Table 3

The fitting results for different intervals of $M^{2}$ in PT with $\Lambda=0.25 \mathrm{GeV}$

\begin{tabular}{|c|c|c|c|c|c|}
\hline Интервал по $M^{2}$, ГэВ & $C_{2}$ (с ошибками), Гэ $\mathrm{B}^{2}$ & $\left\langle\alpha_{s} G G\right\rangle / \pi, \Gamma э \mathrm{~B}^{4}$ & $\chi_{\min }^{2}$ & (для $C_{2}=0$ ) & $\begin{array}{c}\text { Антикорреляция } \\
\text { между } C_{2} \\
\text { и глюонным конденсатом }\end{array}$ \\
\hline$[10 / 20,160 / 20]$ & $-0,093 \mp 0,054$ & $0,025 \pm 0,008$ & 0,758 & 3 & $-0,153 C_{2}+0,011$ \\
\hline$[11 / 20,120 / 20]$ & $-0,076 \mp 0,052$ & $0,023 \pm 0,008$ & 0,553 & 3 & $-0,154 C_{2}+0,011$ \\
\hline$[12 / 20,100 / 20]$ & $-0,065 \mp 0,052$ & $0,021 \pm 0,008$ & 0,406 & 2 & $-0,154 C_{2}+0,011$ \\
\hline$[13 / 20,90 / 20]$ & $-0,058 \mp 0,053$ & $0,020 \pm 0,008$ & 0,323 & 2 & $-0,154 C_{2}+0,011$ \\
\hline$[14 / 20,80 / 20]$ & $-0,052 \mp 0,053$ & $0,019 \pm 0,008$ & 0,265 & 1 & $-0,155 C_{2}+0,011$ \\
\hline$[15 / 20,70 / 20]$ & $-0,047 \mp 0,052$ & $0,018 \pm 0,008$ & 0,212 & 1 & $-0,155 C_{2}+0,011$ \\
\hline$[16 / 20,60 / 20]$ & $-0,042 \mp 0,051$ & $0,017 \pm 0,008$ & 0,156 & 1 & $-0,155 C_{2}+0,011$ \\
\hline$[17 / 20,50 / 20]$ & $-0,037 \mp 0,048$ & $0,016 \pm 0,007$ & 0,097 & 1 & $-0,156 C_{2}+0,011$ \\
\hline$[18 / 20,40 / 20]$ & $-0,032 \mp 0,044$ & $0,016 \pm 0,007$ & 0,041 & 1 & $-0,156 C_{2}+0,011$ \\
\hline$[19 / 20,30 / 20]$ & $-0,027 \mp 0,036$ & $0,015 \pm 0,006$ & 0,005 & 1 & $-0,156 C_{2}+0,011$ \\
\hline
\end{tabular}

Таблица 4

Результаты фитирования на различных интервалах по $M^{2}$ в АТВ при $\Lambda=0,25$ ГэВ

Table 4

The fitting results for different intervals of $M^{2}$ in APT with $\Lambda=0.25 \mathrm{GeV}$

\begin{tabular}{|c|c|c|c|c|c|}
\hline Интервал по $M^{2}$, ГэВ & $\tilde{C}_{2}$ (с ошибками), ГэВ & $\left\langle\alpha_{s} G G / \pi, \Gamma э \mathrm{~B}^{4}\right.$ & $\chi_{\min }^{2}$ & $\begin{array}{c}\text { Антикорреляция } \\
\left.\text { (для } \tilde{C}_{2}=0\right)\end{array}$ & $\begin{array}{c}\text { между } \tilde{C}_{2} \text { и глюонным } \\
\text { конденсатом }\end{array}$ \\
\hline$[10 / 20,160 / 20]$ & $-0,067 \mp 0,053$ & $0,026 \pm 0,008$ & 0,723 & 2 & $-0,159 \tilde{C}_{2}+0,016$ \\
\hline$[11 / 20,120 / 20]$ & $-0,048 \mp 0,053$ & $0,023 \pm 0,008$ & 0,508 & 1 & $-0,159 \tilde{C}_{2}+0,016$ \\
\hline$[12 / 20,100 / 20]$ & $-0,036 \mp 0,054$ & $0,021 \pm 0,009$ & 0,368 & 1 & $-0,159 \tilde{C}_{2}+0,016$ \\
\hline$[13 / 20,90 / 20]$ & $-0,028 \mp 0,057$ & $0,020 \pm 0,009$ & 0,296 & 1 & $-0,159 \tilde{C}_{2}+0,016$ \\
\hline$[14 / 20,80 / 20]$ & $-0,022 \mp 0,058$ & $0,019 \pm 0,009$ & 0,244 & 1 & $-0,159 \tilde{C}_{2}+0,016$ \\
\hline$[15 / 20,70 / 20]$ & $-0,017 \mp 0,059$ & $0,018 \pm 0,009$ & 0,195 & 1 & $-0,159 \tilde{C}_{2}+0,016$ \\
\hline$[16 / 20,60 / 20]$ & $-0,012 \mp 0,058$ & $0,017 \pm 0,009$ & 0,142 & 1 & $-0,159 \tilde{C}_{2}+0,016$ \\
\hline$[17 / 20,50 / 20]$ & $-0,006 \mp 0,055$ & $0,017 \pm 0,009$ & 0,086 & 1 & $-0,160 \tilde{C}_{2}+0,016$ \\
\hline$[18 / 20,40 / 20]$ & $-0,000 \mp 0,051$ & $0,016 \pm 0,008$ & 0,035 & 1 & $-0,160 \tilde{C}_{2}+0,016$ \\
\hline$[19 / 20,30 / 20]$ & $0,006 \mp 0,045$ & $0,015 \pm 0,007$ & 0,004 & 1 & $-0,160 \tilde{C}_{2}+0,016$ \\
\hline
\end{tabular}




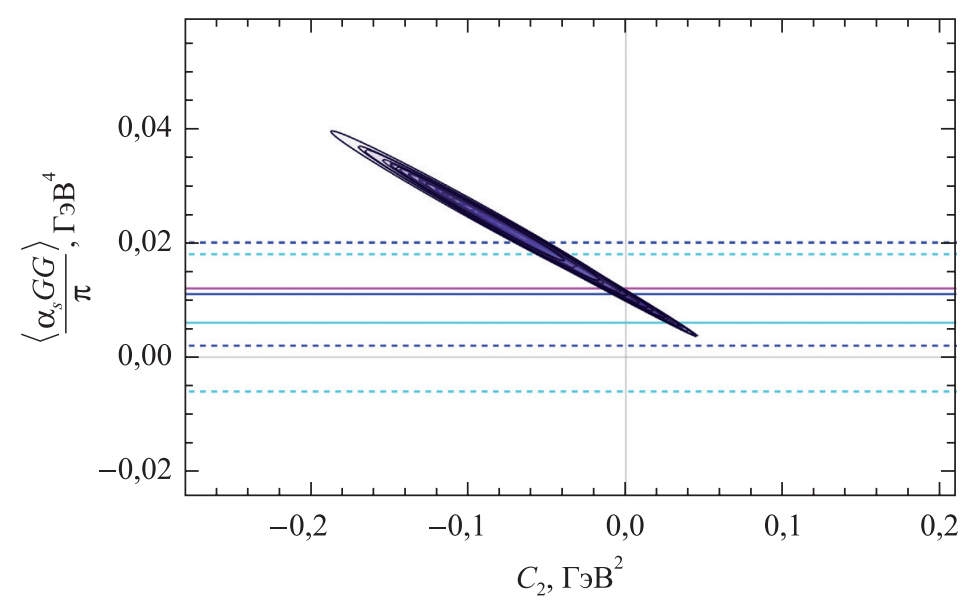

Puc. 2. Антикорреляция короткой струны и глюонного конденсата в ТВ при $\Lambda=0,25$ ГэВ Fig. 2. The (anti)correlation between short string and gluon condensate in PT at $\Lambda=0.25 \mathrm{GeV}$

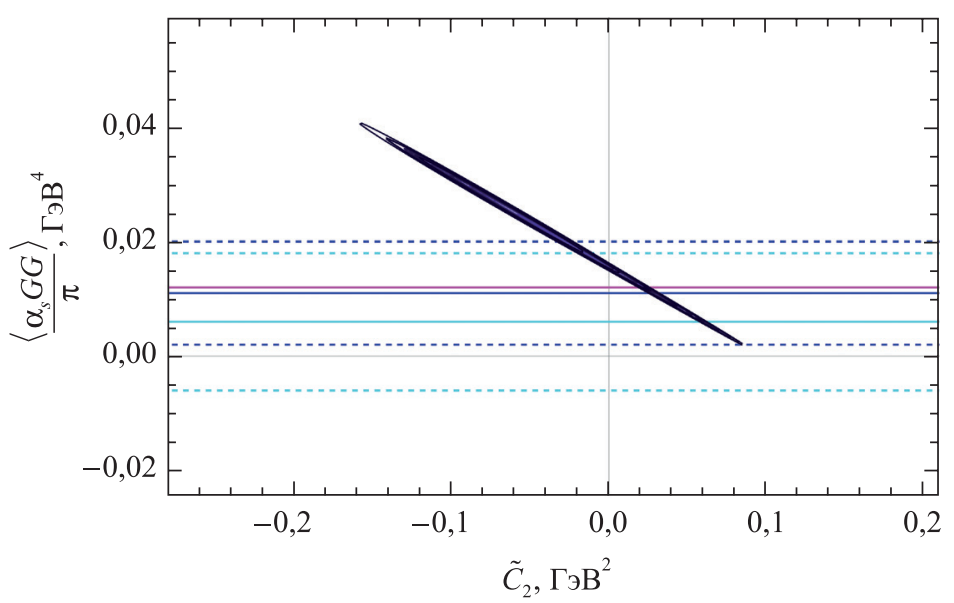

Puc. 3. Антикорреляция короткой струны и глюонного конденсата в АТВ при $\Lambda=0,25$ ГэВ Fig. 3. The (anti)correlation between short string and gluon condensate in APT at $\Lambda=0.25 \mathrm{GeV}$

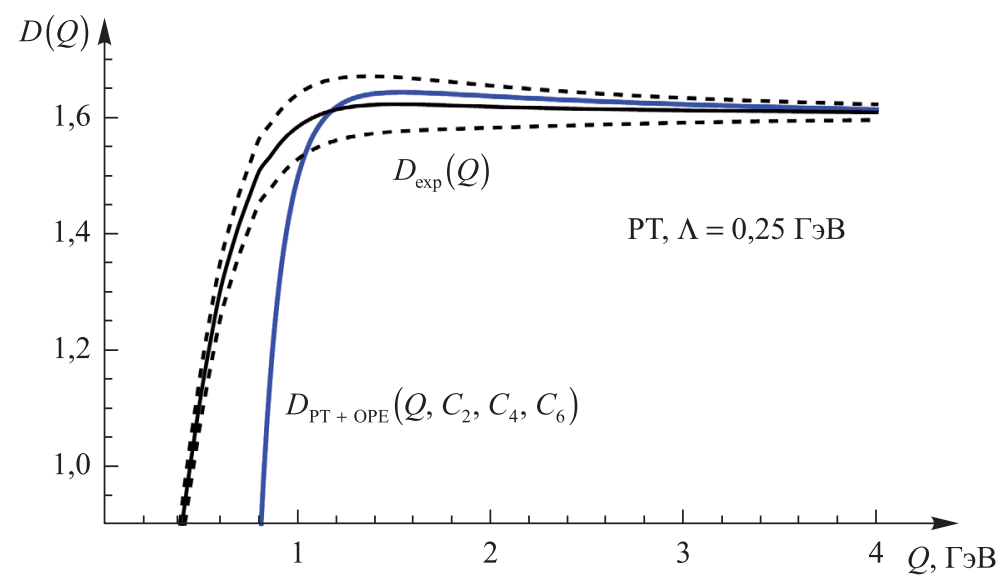

Puc. 4. Стандартная зависимость функции Адлера от $Q$ с полученными значениями $C_{2}$ и $C_{4}$ (синяя кривая) в сравнении с дисперсионной формулой (черная кривая)

с учетом доминирующей систематической ошибки (статистическая пренебрежимо мала)

Fig. 4. The standard dependence of Adler function on $Q$ for PT

with received values of $C_{2}$ and $C_{4}$ (blue) and dispersion curve (black)

with taking into account dominant systematic error (statistic error is negligible) 


\section{Заключение}

Построена модель для $R$-отношения $\mathrm{e}^{+} \mathrm{e}^{-}$-аннигиляции, достаточно хорошо описывающая экспериментальные данные. С помощью этой модели получена функция Адлера и ее преобразование Бореля. Извлечены параметры операторного разложения, а именно вклад короткой струны $\left(C_{2}\right)$ и глюонного конденсата (пропорционального $C_{4}$ ). Подтверждено сделанное ранее наблюдение их сильной антикорреляции. Вклад короткой струны лучше совместим с нулем при использовании АТВ.

Построена стандартная зависимость функции Адлера от $Q$ с использованием найденных значений $C_{2}$ и $C_{4}$. Кривая, полученная с помощью операторного разложения, находится в разумном согласии с дисперсионной кривой при $Q>1$ ГэВ.

\section{Библиографические ссылки}

1. Shifman MA, Vainshtein AI, Zakharov VI. QCD and resonance physics. Theoretical foundations. Nuclear Physics B. 1979; 147(5):385-447. DOI: 10.1016/0550-3213(79)90022-1.

2. Shifman MA, Vainshtein AI, Zakharov VI. QCD and resonance physics. Applications. Nuclear Physics B. 1979;147(5): 448-518. DOI: 10.1016/0550-3213(79)90023-3.

3. Chetyrkin KG, Narison S, Zakharov VI. Short-distance tachyonic gluon mass and 1/Q ${ }^{2}$ corrections Nuclear Physics B. 1999; 550(1-2):353-374. DOI: 10.1016/S0550-3213(99)00167-4.

4. Narison S, Zakharov VI. Duality between QCD perturbative series and power corrections. Physics Letters B. 2009;679(4): 355-361. DOI: 10.1016/j.physletb.2009.07.060.

5. Bodenstein S, Dominguez CA, Eidelman SI, Spiesberger H, Schilcher K. Confronting electron-positron annihilation into hadrons with QCD: an operator product expansion analysis. Journal of High Energy Physics. 2012;2012:39. DOI: 10.1007/JHEP01(2012)039.

6. Eichten E, Gottfried K, Kinoshita T, Kogut J, Lane KD, Yan T-M. Spectrum of charmed quark-antiquark bound states. Physical Review Letters. 1975;34(6):369. Erratum: [Physical Review Letters. 1276(1976);36]. DOI: 10.1103/PhysRevLett.34.369.

7. Kataev AL, Parente G, Sidorov AV. Improved fits to the $x F_{3}$ CCFR data at the next-to-next-to-leading order and beyond. Physics of Particles and Nuclei. 2007;38(6):827. DOI: 10.1134/S1063779607060068.

8. Khandramai VL, Pasechnik RS, Shirkov DV, Solovtsova OP, Teryaev OV. Four-loop QCD analysis of the Bjorken sum rule. Physics Letters B. 2012;706(4-5):340-344. DOI: 10.1016/j.physletb.2011.11.023.

9. Spiesberger H. An operator product expansion analysis of $\mathrm{e}^{+} \mathrm{e}^{-}$annihilation data. Modern Physics Letters A. 2013;28(25): 1360009. DOI: $10.1142 / \mathrm{S} 0217732313600092$.

10. Solovtsova OP. Manifestation of quark-hadron duality in $\mathrm{e}^{+} \mathrm{e}^{-}$annihilation into hadrons. Nonlinear phenomena in complex systems. 2014;17(4):445-447.

11. Shirkov DV, Solovtsov IL. Analytic Model for the QCD Running Coupling with Universal $\bar{\alpha}_{s}(0)$ Value. Physical Review Letters. 1997;79:1209. DOI: 10.1103/PhysRevLett.79.1209.

12. Milton KA, Solovtsov IL. Analytic perturbation theory in QCD and Schwinger's connection between the $\beta$ function and the spectral density. Physical Review D. 1997;55:5295. DOI: 10.1103/PhysRevD.55.5295.

13. Shirkov DV, Solovtsov IL. Ten years of the analytic perturbation theory in QCD. Theoretical and Mathematical Physics. 2007; 150(1):152-176. DOI: $10.4213 / \mathrm{tmf5971}$

14. Milton KA, Solovtsova OP. Analytic perturbation theory: a new approach to the analytic continuation of the strong coupling constant $\alpha_{S}$ into the timelike region. Physical Review D. 1998;57:5402. DOI: 10.1103/PhysRevD.57.5402.

15. Pasechnik RS, Shirkov DV, Teryaev OV, Solovtsova OP, Khandramai VL. Nucleon spin structure and perturbative QCD frontier on the move. Physical Review D. 2010;81(1):016010. DOI: 10.1103/PhysRevD.81.016010.

16. Sidorov AV, Solovtsova OP. The QCD Analysis of $x F_{3}$ structure function based on the analytic approach. Nonlinear Phenomena in Complex Systems. 2013;16(4):397-402.

17. Sidorov AV, Solovtsova OP. QCD analysis of the $\mathrm{F}_{3}$ structure function based on inverse Mellin transform in analytic perturbation theory. Physics of Particles and Nuclei Letters. 2017;14(1):1-8. DOI: 10.1134/S154747711701023X.

18. Barkov LM, Chilingarov AG, Eidelman SI, Khazin BI, Lelchuk MYu, Okhapkin VS, et al. Electromagnetic pion form factor in the timelike region. Nuclear Physics B. 1985;256:365-384. DOI: 10.1016/0550-3213(85)90399-2.

19. Aubert B, Barate R, Boutigny D, Couderc F, Karyotakis Y, Lees JP, et al. (BABAR Collaboration). $\mathrm{e}^{+} \mathrm{e}^{-} \rightarrow \pi^{+} \pi^{-} \pi^{+} \pi^{-}$, $K^{+} K^{-} \pi^{+} \pi^{-}$, and $K^{+} K^{-} K^{+} K^{-}$cross sections at center-of-mass energies $0.5-4.5 \mathrm{GeV}$ measured with initial-state radiation. Physical Review D. 2005;71:052001. DOI: 10.1103/PhysRevD.71.052001.

20. Whalley MR. A compilation of data on hadronic total cross sections in $\mathrm{e}^{+} \mathrm{e}^{-}$interactions. Journal of Physics G: Nuclear and Particle Physics. 2003;29(12A):A1-A133. DOI: 10.1088/0954-3899/29/12A/R01.

21. Kurdadze LM, Lel'Chuk MYu, Pakhtusova EV, Sidorov VA, Skrinskiľ AN, Chilingarov AG, et al. Study of the reaction $\mathrm{e}^{+} \mathrm{e}^{-} \rightarrow \pi^{+} \pi^{-} \pi^{0} \pi^{0}$ at (2e) up to $1.4 \mathrm{GeV}$. Journal of Experimental and Theoretical Physics Letters. 1986;43:643-645.

22. Dolinsky SI, Druzhinin VP, Dubrovin MS, Golubev VB, Ivanchenko VN, Pakhtusova EV, et al. Summary of experiments with the neutral detector at the $\mathrm{e}^{+} \mathrm{e}^{-}$storage ring VEPP-2M. Physics Reports. 1991;202(3):99-170. DOI: 10.1016/0370-1573(91)90127-8.

23. Ачасов МН, Бару СЕ, Белобородов КИ, Бердюгин АВ, Боженок АВ, Богданчиков АГ и др. Исследование проиессов $\mathrm{e}^{+} \mathrm{e}^{-} \rightarrow 4 \pi$ в области энергии 0,98-1,38 ГэВ с детектором СНД. Новосибирск: Институт ядерной физики им. Г. И. Будкера CO PAH; 2001.

24. Bisello B, Busetto G, Castro A, Nigro M, Pescara L, Sartori P, et al. (DM2 Collaboration). DM2 results on $\mathrm{e}^{+} \mathrm{e}^{-}$annihilation into multihadrons in the 1350-2400 MeV energy range. In: Phua KK, Yamaguchi Y, editors. Rochester Conference on High-Energy Physics. $25^{\text {th }}$ International Conference on High-Energy Physics; 1990 August 2-8; Singapore, Singapore. Singapore: World Scientific; 1991. LAL-90-35. 
25. Cosme G, Dudelzak B, Grelaud B, Jean-Marie B, Jullian S, Lalanne D, et al. Hadronic cross sections study in $\mathrm{e}^{+} \mathrm{e}^{-}$collisions from 1.350 GeV to 2.125 GeV. Nuclear Physics B. 1979;152(2):215-231. DOI: 10.1016/0550-3213(79)90100-7.

26. Aubert B, Barate R, Boutigny D, Couderc F, Karyotakis Y, Lees JP, et al. The $\mathrm{e}^{+} \mathrm{e}^{-} \rightarrow 3\left(\pi^{+} \pi^{-}\right), 2\left(\pi^{+} \pi^{-} \pi^{0}\right)$ and $K^{+} K^{-} 2\left(\pi^{+} \pi^{-}\right)$ cross sections at center-of-mass energies from production threshold to $4.5 \mathrm{GeV}$ measured with initial-state radiation. Physical Review D. 2006;73(5):052003. DOI: 10.1103/PhysRevD.73.052003.

27. Gounaris GJ, Sakurai JJ. Finite-width corrections to the vector-meson-dominance prediction for $\rho \rightarrow \mathrm{e}^{+} \mathrm{e}^{-}$. Physical Review Letters. 1968;21:244. DOI: 10.1103/PhysRevLett.21.244.

28. Olive KA, Agashe K, Amsler C, Antonelli M, Arguin J-F, Asner DM, et al. (Particle Data Group). Review of particle physics. Chinese Physics C. 2014;38(9):090001. DOI: 10.1088/1674-1137/38/9/090001.

29. Geshkenbein BV, Ioffe BL, Zyablyuk KN. Check of QCD based on the $\tau$-decay data analysis in the complex $q^{2}$ plane. Physical Review D. 2001;64(9):093009. DOI: 10.1103/PhysRevD.64.093009.

30. Kozhevnikova ME, Oganesian AG, Teryaev OV. Search for short strings in $\mathrm{e}^{+} \mathrm{e}^{-}$-annihilation. EPJ Web Conferences. 2017; 138:02006. DOI: 10.1051/epjconf/201713802006.

31. Ioffe BL, Zyablyuk KN. Gluon condensate in charmonium sum rules with three-loop corrections. European Physical Journal C. 2003;27(2):229-241. DOI: 10.1140/epjc/s2002-01099-8.

\section{References}

1. Shifman MA, Vainshtein AI, Zakharov VI. QCD and resonance physics. Theoretical foundations. Nuclear Physics B. 1979; 147(5):385-447. DOI: 10.1016/0550-3213(79)90022-1.

2. Shifman MA, Vainshtein AI, Zakharov VI. QCD and resonance physics. Applications. Nuclear Physics B. 1979;147(5): 448-518. DOI: 10.1016/0550-3213(79)90023-3.

3. Chetyrkin KG, Narison S, Zakharov VI. Short-distance tachyonic gluon mass and $1 / \mathrm{Q}^{2}$ corrections Nuclear Physics B. 1999; 550(1-2):353-374. DOI: 10.1016/S0550-3213(99)00167-4

4. Narison S, Zakharov VI. Duality between QCD perturbative series and power corrections. Physics Letters B. 2009;679(4): 355-361. DOI: 10.1016/j.physletb.2009.07.060

5. Bodenstein S, Dominguez CA, Eidelman SI, Spiesberger H, Schilcher K. Confronting electron-positron annihilation into hadrons with QCD: an operator product expansion analysis. Journal of High Energy Physics. 2012;2012:39. DOI: 10.1007/JHEP01(2012)039.

6. Eichten E, Gottfried K, Kinoshita T, Kogut J, Lane KD, Yan T-M. Spectrum of charmed quark-antiquark bound states. Physical Review Letters. 1975;34(6):369. Erratum: [Physical Review Letters. 1276(1976);36]. DOI: 10.1103/PhysRevLett.34.369.

7. Kataev AL, Parente G, Sidorov AV. Improved fits to the $x F_{3}$ CCFR data at the next-to-next-to-leading order and beyond. Physics of Particles and Nuclei. 2007;38(6):827. DOI: 10.1134/S1063779607060068.

8. Khandramai VL, Pasechnik RS, Shirkov DV, Solovtsova OP, Teryaev OV. Four-loop QCD analysis of the Bjorken sum rule. Physics Letters B. 2012;706(4-5):340-344. DOI: 10.1016/j.physletb.2011.11.023.

9. Spiesberger H. An operator product expansion analysis of $\mathrm{e}^{+} \mathrm{e}^{-}$annihilation data. Modern Physics Letters A. 2013;28(25): 1360009. DOI: 10.1142/S0217732313600092.

10. Solovtsova OP. Manifestation of quark-hadron duality in $\mathrm{e}^{+} \mathrm{e}^{-}$annihilation into hadrons. Nonlinear phenomena in complex systems. 2014;17(4):445-447.

11. Shirkov DV, Solovtsov IL. Analytic Model for the QCD Running Coupling with Universal $\bar{\alpha}_{s}(0)$ Value. Physical Review Letters. 1997;79:1209. DOI: 10.1103/PhysRevLett.79.1209.

12. Milton KA, Solovtsov IL. Analytic perturbation theory in QCD and Schwinger's connection between the $\beta$ function and the spectral density. Physical Review D. 1997;55:5295. DOI: 10.1103/PhysRevD.55.5295.

13. Shirkov DV, Solovtsov IL. Ten years of the analytic perturbation theory in QCD. Theoretical and Mathematical Physics. 2007; 150(1):152-176. DOI: $10.4213 / \mathrm{tmf5971.}$

14. Milton KA, Solovtsova OP. Analytic perturbation theory: a new approach to the analytic continuation of the strong coupling constant $\alpha_{S}$ into the timelike region. Physical Review D. 1998;57:5402. DOI: 10.1103/PhysRevD.57.5402.

15. Pasechnik RS, Shirkov DV, Teryaev OV, Solovtsova OP, Khandramai VL. Nucleon spin structure and perturbative QCD frontier on the move. Physical Review D. 2010;81(1):016010. DOI: 10.1103/PhysRevD.81.016010.

16. Sidorov AV, Solovtsova OP. The QCD Analysis of $x F_{3}$ structure function based on the analytic approach. Nonlinear Phenomena in Complex Systems. 2013;16(4):397-402.

17. Sidorov AV, Solovtsova OP. QCD analysis of the $\mathrm{F}_{3}$ structure function based on inverse Mellin transform in analytic perturbation theory. Physics of Particles and Nuclei Letters. 2017;14(1):1-8. DOI: 10.1134/S154747711701023X.

18. Barkov LM, Chilingarov AG, Eidelman SI, Khazin BI, Lelchuk MYu, Okhapkin VS, et al. Electromagnetic pion form factor in the timelike region. Nuclear Physics B. 1985;256:365-384. DOI: 10.1016/0550-3213(85)90399-2.

19. Aubert B, Barate R, Boutigny D, Couderc F, Karyotakis Y, Lees JP, et al. (BABAR Collaboration). $\mathrm{e}^{+} \mathrm{e}^{-} \rightarrow \pi^{+} \pi^{-} \pi^{+} \pi^{-}$, $K^{+} K^{-} \pi^{+} \pi^{-}$, and $K^{+} K^{-} K^{+} K^{-}$cross sections at center-of-mass energies $0.5-4.5 \mathrm{GeV}$ measured with initial-state radiation. Physical Review D. 2005;71:052001. DOI: 10.1103/PhysRevD.71.052001.

20. Whalley MR. A compilation of data on hadronic total cross sections in $\mathrm{e}^{+} \mathrm{e}^{-}$interactions. Journal of Physics G: Nuclear and Particle Physics. 2003;29(12A):A1-A133. DOI: 10.1088/0954-3899/29/12A/R01.

21. Kurdadze LM, Lel'Chuk MYu, Pakhtusova EV, Sidorov VA, Skrinskiǐ AN, Chilingarov AG, et al. Study of the reaction $\mathrm{e}^{+} \mathrm{e}^{-} \rightarrow \pi^{+} \pi^{-} \pi^{0} \pi^{0}$ at (2e) up to 1.4 GeV. Journal of Experimental and Theoretical Physics Letters. 1986;43:643-645.

22. Dolinsky SI, Druzhinin VP, Dubrovin MS, Golubev VB, Ivanchenko VN, Pakhtusova EV, et al. Summary of experiments with the neutral detector at the $\mathrm{e}^{+} \mathrm{e}^{-}$storage ring VEPP-2M. Physics Reports. 1991;202(3):99-170. DOI: 10.1016/0370-1573(91)90127-8.

23. Achasov MN, Baru SE, Beloborodov KI, Berdyugin AV, Bozhenok AV, Bogdanchikov AG, et al. $\mathrm{e}^{+} \mathrm{e}^{-} \rightarrow 4 \pi$ processes investigation in the energy range $0.98-1.38 \mathrm{GeV}$ with SND detector. Novosibirsk: Budker Institute of Nuclear Physics of Siberian Branch Russian Academy of Sciences; 2001. Russian. 
24. Bisello B, Busetto G, Castro A, Nigro M, Pescara L, Sartori P, et al. (DM2 Collaboration). DM2 results on $\mathrm{e}^{+} \mathrm{e}^{-}$annihilation into multihadrons in the 1350-2400 MeV energy range. In: Phua KK, Yamaguchi Y, editors. Rochester Conference on High-Energy Physics. $25^{\text {th }}$ International Conference on High-Energy Physics; 1990 August 2-8; Singapore, Singapore. Singapore: World Scientific; 1991. LAL-90-35.

25. Cosme G, Dudelzak B, Grelaud B, Jean-Marie B, Jullian S, Lalanne D, et al. Hadronic cross sections study in $\mathrm{e}^{+} \mathrm{e}^{-}$collisions from 1.350 GeV to $2.125 \mathrm{GeV}$. Nuclear Physics B. 1979;152(2):215-231. DOI: 10.1016/0550-3213(79)90100-7.

26. Aubert B, Barate R, Boutigny D, Couderc F, Karyotakis Y, Lees JP, et al. The $\mathrm{e}^{+} \mathrm{e}^{-} \rightarrow 3\left(\pi^{+} \pi^{-}\right), 2\left(\pi^{+} \pi^{-} \pi^{0}\right)$ and $K^{+} K^{-} 2\left(\pi^{+} \pi^{-}\right)$ cross sections at center-of-mass energies from production threshold to $4.5 \mathrm{GeV}$ measured with initial-state radiation. Physical Review D. 2006;73(5):052003. DOI: 10.1103/PhysRevD.73.052003.

27. Gounaris GJ, Sakurai JJ. Finite-width corrections to the vector-meson-dominance prediction for $\rho \rightarrow \mathrm{e}^{+} \mathrm{e}^{-}$. Physical Review Letters. 1968;21:244. DOI: 10.1103/PhysRevLett.21.244.

28. Olive KA, Agashe K, Amsler C, Antonelli M, Arguin J-F, Asner DM, et al. (Particle Data Group). Review of particle physics. Chinese Physics C. 2014;38(9):090001. DOI: 10.1088/1674-1137/38/9/090001.

29. Geshkenbein BV, Ioffe BL, Zyablyuk KN. Check of QCD based on the $\tau$-decay data analysis in the complex $q^{2}$ plane. Physical Review D. 2001;64(9):093009. DOI: 10.1103/PhysRevD.64.093009.

30. Kozhevnikova ME, Oganesian AG, Teryaev OV. Search for short strings in $\mathrm{e}^{+} \mathrm{e}^{-}$-annihilation. EPJ Web Conferences. 2017; 138:02006. DOI: 10.1051/epjconf/201713802006.

31. Ioffe BL, Zyablyuk KN. Gluon condensate in charmonium sum rules with three-loop corrections. European Physical Journal C. 2003;27(2):229-241. DOI: 10.1140/epjc/s2002-01099-8. 